\title{
Retraction Note: Marine biological ecological environment monitoring based on complex dynamic network model
}

\author{
Yandong $\mathrm{Yu}^{1} \cdot$ Yang $\mathrm{Nie}^{2} \cdot$ Yuge Yao $^{1} \cdot$ Lili Jing $^{2}$
}

Published online: 7 December 2021

C) Saudi Society for Geosciences 2021

Retraction Note: Arabian Journal of Geosciences (2021) 14: 874 https://doi.org/10.1007/s12517-021-07227-8

The Editor-in-Chief and the Publisher have retracted this article because the content of this article is nonsensical. The peer review process was not carried out in accordance with the Publisher's peer review policy. Author Lili Jing disagrees with this retraction. Authors Yandong Yu, Yang Nie and Yuge Yao have not responded to correspondence regarding this retraction.

Lili Jing

jnsyyyd@163.com

Yandong Yu

cfssyyd@163.com

Yang Nie

nieyangwork@163.com

Yuge Yao

yaoyuge@163.com

1 Department of Computer, Institute of Software, Key

Laboratory of Internet of Things at School Level, Jining

Normal University, Wulanchabu 012000, China

2 Department of Physics, Key Laboratory of Internet

of Things at School Level, JiningNormal University,

Wulanchabu 012000, China 